more expert knowledge and treatment is called for. In a certain proportion of cases the technique of Freudian psycho-analysis will hold out the best chances of cure. Those patients who, for any reason, cannot be adequately treated by the general practitioner can be referred to one or other of the many clinics which exist for this purpose. It will, however, add greatly to the interest of general practice work if the doctors themselves further their psychological knowledge and learn to apply it themselves.

\section{Concluding Remarḱs}

It is not feasible here to speak of the types of cases to which psychotherapy should apply. In some degree it may be said that every patient will need some of its applications, even if it be only that of instilling cheerfulness, hope, and courage. It is in this way that the personality of the physician, his bedside manner, and the atmosphere he creates in his consulting room mean so much. Conan Doyle told us a short story of two youthful medicos, lately qualified and well versed in the use of all the latest medical knowledge and appliances, who set up in a small community as partners. The other doctor there was an old man with a lovable personality, who had been there for many years, and who was by no means up to date in his work. After their courtesy visit the budding practitioners laughed among themselves at his age and ignorance, pitied his patients, and came to the conclusion that before long he would not have many left when their academic superiority became recognized by the inhabitants. Soon afterwards, on the outbreak of an epidemic of influenza, one of the young doctors was struck down. As he lay in bed feeling very sorry for himself the thought of the scientific attitude of his partner at his bedside had no attraction for him, whereas the presence of the old doctor, he felt, would bring peace and comfort. $\mathrm{He}$ therefore sent for the latter, but found that his partner, who had been taken ill as well, had also sent for the old man they had both so much derided. The moral is obvious.

Though it is in the realm of the psychoneuroses that psychotherapy especially applies, it must be borne in mind that psychogenic factors so frequently have bodily manifestations. The old bugaboo of the relations existing between mind and body must be relegated to the dust-heap. We must regard body and mind as simply different aspects of an individual who is a psychosomatic unity. All the emotions have their physical expressions. It is therefore important in every patient to view the individual as a whole and take into careful consideration possible psychological factors as well as bodily ones. In time experience will teach you that the mental element enters far more into disease than you ever dreamt of. The increased heart-beat may be due to a secret fear; the lump in the throat the result of an insult that cannot be swallowed ; the obstinate feeling of nausea or vomiting may be the sequel of the inability to digest a psychic situation with a resulting buried complex of disgust and revulsion. We do not for nothing speak of a sickening horror or of having a nasty taste in our mouth after reading something of a disagreeable nature. All our emotional feelings may be expressed somatically, and if long continued may bring about definite organic disease. Superficial inquiries, at any rate, should always be made into the emotional life, into the possibility of there being ungratified instinctual yearnings; and the sphere of sexuality must not be neglected. Your patient must be viewed not as a " case," but as a human personality that somehow or other is maladapting to his environment. In dealing with the human body never forget the human heart. With the earnest desire to understand, and with a comparatively small knowledge of medical psychology, the practitioner will be surprised at the fresh light that will be thrown on many recalcitrant symptoms.

Now a word as to prognosis. Commonly enough too much is expected of the psychotherapist. Just as we come across people with poorly developed chests who will always be liable to coughs and who break down under respiratory strain, so we meet with a constitutionally poor mental soil that will ever be apt to find it difficult to withstand the stresses of life without developing symptoms of maladaptation. Whatever method we adopt in psychotherapy we must be prepared to face the fact that we cannot make some individuals normal, but perhaps can alleviate to some extent and render life more bearable. The less we use the word " cure " in psychotherapeutic practice the better. On the other hand, at times brilliant success may be achieved. Those of you who are sufficiently interested to learn more of that hitherto neglected subject-psychological medicine-will find it a most engrossing study, and one that will greatly enhance your usefulness as a physician.

\section{INADEQUATE IMMOBILIZATION AND NON-UNION OF FRACTURES * BY}

\section{R. WATSON JONES, M.CH.ORTH., F.R.C.S.}

LECTURER IN ORTHOPAEDIC PATHOLOGY, LIVERPCOL UNIVERSITY : ASSISTANT SURGEON AND IN CHARGE OF ORTHOPAEDICS, LIVERPOOL ROYAL INFIRIIARY

The crippling disabilities which at one time resulted from excessive immobilization of joints in the treatment of fractures entirely justify the recent wave of enthusiasm for early mobilization and functional activity. The essential principle of modern treatment is complete immobilization of the fracture, with at the same time active movement of every joint which need not be immobilized. When this principle is observed massage treatment becomes unnecessary, incapacity periods are reduced, and many permanent disabilities are avoided. But there is some danger of undue enthusiasm and of misrepresenta tion of the principle, so that the mobilization of joirts is allowed to interfere with the immobilization of the fracture. It must be recognized that complete immobility of the fracture is a primary necessity, that there must be no mobilization except in joints which need not be immobilized, and that mobilization should be the result of the patient's own active exercise and not of the passive manipulations of the masseuse.

A fracture of both forearm bones was recently plated by a distinguished surgeon, and from the time of operation the arm was taken daily from its splints for two hours of massage and passive movement. The conc cant strain on the fractures had not only caused non-union, but had produced painful spasm of the muscles and stiff.ness of the joints, which was even greater than if there had been no movement at all. When the fractures were completely immobilized by a plaster cast active finger movements could be practised without pain and improvement in the range was immediately observed.

Such gross misapplication of the principles of early functional activity in fracture treatment is rare. Unfortunately, however, equally disastrous results are not uncommonly seen when the principles have been misapplied in a less obvious manner. In one group of cases splints or plaster have been inexpertly applied so that the fracture has not actually been immobilized at all. Angulatory movement may have been completely con-

* A paper read in Manchester on March 8th, 1934, at a joint meeting of the Liverpool Medical Institution and the Manchester Medical Society. 
trolled, but the fact that rotatory torsion strains inhibit union just as certainly has been overlooked. In the second group of cases complete immobility has been maintained for six or eight weeks, but the joints have then been mobilized before the fracture is sufficiently consolidated. These two factors-incomplete immobilization and immobilization for too short a period-are the factors responsible for delayed union and non-union, whether in the shaft of a long bone such as the tibia, the humerus, or the ulna, in the neck of the femur, or in the carpal scaphoid bone.

The many theories of inadequate blood supply, impaired nutrition, inaccurate apposition of fragments, failure of impaction, the inhibitory action of synovial fluid, and the absence of blood clot are of no real significance. The only important cause of non-union is inadequate immobilization.

\section{Hyperaemic Bone Decalcification and Non-union}

Recently acquired knowledge of the calcium content of bone in relation to its vascularity provides the pathological basis for these clinical observations. ${ }^{1}$ No longer are we confused by the suggestion that although the decalcification of infected bone is obviously the result of an increased blood supply, the decalcification of fractured bone, such as the fractured neck of the femur, is due to impairment of its blood supply. Hyperaemia of bone always results in decalcification, whether the blood supply is increased by injury, by infection, or by the growth of a neoplasm. Increased calcification or sclerosis, on the other hand, is the result of impairment of blood supply, as may be seen in syphilitic periostitis and Charcot's disease, where there is endarteritis obliterans, or in the late stages of osteomyelitis, where the early infective hyperaemia has been replaced by the ischaemia of reparative fibrosis.

The same hyperaemic decalcification and ischaemic recalcification may be observed in fractures. The initial traumatic hyperaemia gives rise to decalcification of the bone ends. So long as the hyperaemia persists, decalcification goes on: not until it has subsided can calcium salts be redeposited in the young connective tissue which has grown between the bone ends to form callus. When, with the final stages of repair and fibrosis, the.blood supply is impaired, the callus undergoes dense calcification with consolidation of the union.

If, on the other hand, the fragments are imperfectly immobilized, the shearing and twisting strains tear the young connective tissue between the fragments. The repeated traumatization gives rise to constantly recurring hyperaemia, and more and more of the bone ends undergo decalcification. A crack becomes a cavity, a linear fracture becomes a gap fracture. When, finally, there is repair and fibrosis there is no continuous bridge of callus to recalcify, sclerosis is observed in the plaque of bone across the concave bone ends, and non-union is established. Even when the fracture has been properly immobilized for several weeks so that the initial hyperaemia has subsided and recalcification has begun, if it is then repeatedly strained before the stage of final dense consolidation the same traumatic hyperaemia is observed. Recalcification ceases at once, decalcification supervenes, and non-union develops.

\section{Fractures of the Carpal Scaphoid}

Fractures of the scaphoid illustrate very clearly the three stages in the life-history of an ununited fracture. Within a few days of injury the crack is so fine that it may be overlooked in radiographs, and, indeed, it may only be possible to suspect it even with the aid of a magnifying lens. But if it is not immobilized the traumatic hyperaemia of movement of the fragments on each other gives rise to decalcification and the appearance of a cyst. This intermediate stage of non-union persists from about one to four or five months after injury, and the fracture will still unite if it is immobilized. In the third stage, reparative fibrosis and ischaemia have caused recalcification ; the "cavity" has partly filled in, but the surfaces of the fragments are dense and sclerosed. Obviously it is now wasted effort to immobilize the fracture without first breaking up the sclerosis, refreshening the fractured surfaces by multiple drilling, and so allowing revascularization of the bone surfaces and of the intervening fibrous tissue.

The last 100 cases of fracture of the carpal scaphoid which were immobilized at the Liverpool Royal Infirmary may be analysed as follows:

One Hundied Fractures of Carpal Scaphoid Bone

\begin{tabular}{l|c|c|c|c}
\hline & $\begin{array}{c}\text { Number } \\
\text { of } \\
\text { Cases }\end{array}$ & $\begin{array}{c}\text { Average } \\
\text { Period of } \\
\text { Inmoliliza- } \\
\text { tion }\end{array}$ & $\begin{array}{c}\text { Number of } \\
\text { Cases of } \\
\text { Non-union }\end{array}$ & $\begin{array}{c}\text { Percentage } \\
\text { Securing } \\
\text { Bony } \\
\text { Union }\end{array}$ \\
\hline$* \begin{array}{c}\text { Recent fractures of waist } \\
\text { and tubercle }\end{array}$ & 66 & 2 months & None & 100 \\
$* \begin{array}{c}\text { Recent fractures of proxi- } \\
\text { mal pole }\end{array}$ & 6 & $5 \frac{1}{2}$ months \\
$+\begin{array}{c}\text { Old fractures of waist } \\
\text { and proximal pole }\end{array}$ & 23 & $7 \frac{1}{2}$ months & 1 & 83 \\
$+\begin{array}{c}\text { Old fractures of waist } \\
\text { with dislocation semi- } \\
\text { lunar }\end{array}$ & 5 & 16 months & 2 & 65 \\
\hline
\end{tabular}

* Recent-less than 1 month old.

+ Old-less than 1 year old (drilling in 10 cases).

Cases older than 1 year (usually with osteoarthritis of the wrist) were not immobilized.

It is evident that in recent fractures, even including the difficult polar fractures, bony union may be secured in 98 per cent. of cases by complete immobilization with an unpadded plaster cast. The average duration of immobilization is little over two months, but occasionally it is necessary, even in recent fractures, to continue for six or twelve months. If in every case immobilization had ceasiod after two months there would have been a high frequency of non-zinion; but by continuing immobilization until the radiograph shows evidence of consolida. tion every one unites by hone. Similatly, when treatment is delayed for several months, it is still possible to secure union in the majority of cases, but drilling is frequently required and it may be necessary to maintain immobiliza. tion for as long as twenty-two months.

These statistics compare most favourably with the results which were at one time secured by the inadequate immobilization of a cock-up splint for a few weeks. With such treatment bony union was the exception rather than the rule.

\section{Fractures of the Neck of the Femur}

In subcapital fractures of the neck of the femur we have another example of a fracture in which apparently trivial movement, especially rotatory movement, is sufficient to perpetuate traumatic hyperaemic decalcification and to delay or to prevent union of the fragments. It is generally believed that a high fracture of the femoral neck is completely immobilized by a plaster spica extending from the toes to the upper chest. Special precaution may have been taken to flex the knee so that the limb cannot rotate within the plaster; but the fact that the upper fragment can rotate is overlooked. Every surgeon who operates on such a case is impressed with the extraordinary mobility of the detached head of the femur. It has no attachments whatever, apart from the ligamentum teres, and it can twist and slide within the acetabulum even when the limb below it is held rigidly immobile. Moreover, in the obese patient it is practically impossible 
to mould the trunk part of the plaster in such a way that the pelvis is prevented from rotating.

It is this constantly repeated rotation movement of the fragments which accounts for the decalcification and absorption of the neck of the femur with non-union which is seen in about 50 per cent. of the cases so treated. Even in the 50 per cent. which do ultimately unite the same decalcification is seen in the shortening of the neck of the femur which almost always occurs, and which proves that the hyperaemia due to the trauma of movement has continued long after the hyperaemia of the initial injury subsided. There is no such thing as a " dry fracture" of the neck of the femur ; the capsule of the joint is always distended with blood. The distal fragment is more vascular than the proximal fragment, and it is the distal fragment (the neck of the femur) which undergoes the greatest decalcification and absorption.

The stainless steel Smith-Petersen nail is made in such a way that the length of the nail prevents angulatory movement, and the three flanges prevent rotatory movement. With the nail driven centrally through the head and neck of the femur immobility is absolute. For the first time in the history of the treatment of fracture of the neck of the femur we have prevented rotational strain and movement, and for the first time we have a method which secures union of the fracture in 90 to 100 per cent. of cases. Moreover, shortening of the neck of the femur from the decalcification of continued movement of the fragments is no longer observed.

I have operated on twenty-eight subcapital fractures of the neck of the femur with one death. Of the twenty cases in which a stainless steel nail was inserted more than twelve months ago, all have united by bone except one, and this single failure was a case in which the nail was removed four months after operation, before there was radiographic evidence of consolidation. Many of these fractures are not firmly united in the accepted period of three or four months, and if immobilization is discontinued at that stage, union may never go on to consolidation. The imperfectly calcified callus cannot withstand the trauma of movement, and it undergoes decalcification with resulting non-union. If it were reasonable to continue the use of a Whitman plaster for a sufficiently long period, it is possible that most high fractures of the neck of the femur would unite despite the mobility which is permitted. But there is a limit to the endurance of old and fragile patients. The Smith-Petersen nail, on the other hand, may be retained for an indefinite period without hardship ; the fact that it affords absolute immobility, and that the immobility is continued for a sufficiently long period, accounts for the difference between bony union in 100 per cent. of cases by this method and in 50 per cent. by the old Whitman method.

\section{Intra-ARticular Arthrodesis of the OSTEOARTHRITIC HIP}

It is remarkable that the analogy between union of fractures of the neck of the femur and consolidation after arthrodesis of the hip-joint has never been drawn. It is generally recognized that high fractures of the neck of the femur only unite in about 50 per cent. of cases if a plaster spica is relied on for immobilization. It is also recognized that after intra-articular arthrodesis of the osteoarthritic hip only $\mathbf{5 0}$ per cent. of cases consolidate. Wherein lies the difference? It is exactly the same problem-the problem of preventing rotational movement of the fragments. If a Smith-Petersen nail is driven through the neck and head of the femur into the pelvis, every such arthrodesis undergoes firm consolidation, and there is no necessity for the complication of an extra. articular graft. The method has been employed in sixteen cases without failure of consolidation in any.

\section{Non-union in Fractures of the Lower Shaft of THE UlNa}

In fractures of the lower shaft of both forearm bones non-union is commonly seen in the ulna, but is practically never seen in the radius, despite the fact that the injuries were sustained simultaneously, and that their treatment was apparently identical. There is no difference in the blood supply of the bones or in their nutrition. Why should one surgeon in the last four years have operated on fourteen cases of ununited fracture of the lower shaft of the ulna, when in every case the radius had united without difficulty? The explanation lies in the fact that pronation and supination of the forearm is a buckethandle movement of the radius round the fixed axis of the ulna. During the seven or eight weeks which is usually considered an adequate period of immobilization both fractures undergo some degree of union; at the same time the inferior radio-ulnar joint becomes stiff. After removal of the splints or plaster, massage is usually instituted, and an attempt is made to restore radio-ulnar movement. But this movement can occur just as easily at the unconsolidated fracture of the ulna as at the stiffened radio-ulnar joint. The treatment resolves itself into frequent torsion strains of the ulnar fracture, causing traumatic hyperaemia, and therefore decalcification of the callus and non-union. In the radius, on the other hand, both fragments swing together, there is no rotatory strain, and the callus continues to calcify until union is consolidated.*

When these fractures are treated from the beginning in forearm gutter splints or a short below-elbow plaster cast (even though it be unpadded) it is obvious that nonunion of the ulna is still more inevitable. Although such a plaster prevents any angulatory displacement it does not prevent rotatory movement of the fragments. The fracture is not immobilized unless there is absolute limitation of radio-ulnar movement by complete fixation of both wrist and elbow. Moreover, this absolute immobility must be continued for three or four months until there is radiographic evidence of consolidation. With such treatment non-union is never seen.

Even after a successful bone-grafting operation complete immobility must be maintained for several months. Two cases which were grafted several years ago were immobilized for ten weeks. The complete plaster was then replaced by a short cast which prevented angulatory movement, but did not completely limit radio-ulnar movement. In the first case subsequent radiographs .showed progressive decalcification of the graft at the level of fracture with recurrence of non-union. In the other, the graft fused firmly with the distal fragment, but the callus between the graft and the proximal fragment decalcified and non-union persisted. In both cases after regrafting, and in all of the other twelve which have been grafted, firm bony union was secured after several months of immobilization. The duration of immobilization was never less than fourteen weeks; in the last case recalcification was so slow that immobility was maintained for eleven months, and there can be no doubt from the radiographs that if movement had been allowed at any time earlier than this non-union would have recurred.

\section{Non-union in Fractures of the Shafts of Other LONg Bones}

The same relation of a stiffened joint to an unconsolidated fracture is seen in fractures of the lower shaft of the humerus. Not infrequently, in the treatment of this fracture, rotation movement is incompletely controlled and union is delayed. But the elbow-joint rapidly stiffens, and if attempts are made to restore mobility to the joint

* The position is exactly analogous to that of the fractured rib, where with every respiration both fragments move together but not on each other. The intercostal muscles and aponeuroses provide a natural protection from shearing and rotatory strains. 
by passive movement the fracture is strained and nonunion persists. Incidentally this is merely one of the complications which are directly attributable to passive movement of the elbow-joint-a procedure which cannot. be defended on any grounds.

Non-union is quite frequently seen in fractures of the shaft of the femur when a Thomas splint has been inexpertly used, so that the extension tapes have rarely been tight and the limb has been allowed to twist and angulate within the splint. The non-union may persist for months, and yet as soon as adequate nursing is available, so that the extensions never slacken even momentarily, the fractures unite.

The belief that the rate of union of a fracture is determined only by its site, and that the correct treatment is to immobilize for a certain number of weeks and then to institute massage treatment, is responsible for many cases of non-union. It is a stock examination question to ask a candidate how long he would immobilize a fracture of the shaft of the tibia, and there can be no doubt that he would receive Iittle credit for the correct reply: "Until it is united." The rate of union varies very considerably in different individuals, and although many fractures unite in eight or ten weeks, it may be several months before union is consolidated. If at any time before the fracture is clinically firm, absolute fixation is interrupted, even by the substitution of walking splints for the complete plaster, union is delayed. If sufficient mobility is allowed the soft callus undergoes decalcification, and in the course of time the fractured surfaces become sclerosed with establishment of non-union.

\section{INFEcted Compound Fractures}

The infected fracture is exactly like the simple fracture, except that the hyperaemia of infection is added to the hyperaemia of injury, so that decalcification is more marked and continues for a longer period. A wide gap may appear between the fragments, but the space is only apparent, and, like the " cyst" in the old fracture of the scaphoid, it is filled with granulation tissue which will recalcify after quiescence of the infection. If perfect immobilization has been maintained by the Winnett Orr no-dressings-plaster method the fracture will unite. If, on the other hand, the treatment of the infection is allowed to interfere with the immobilization, the fracture fails to unite. The application of the Winnett Orr method is of far greater importance in the treatment of infected fractures than in the treatment of osteomyelitis. However comminuted the fracture may be, however serious the infection, and however extensive the destruction of skin, the use of this method avoids the necessity for amputation in almost every case except that in which the main artery is injured.

It is in the terminal stages of the infection that immobility is of the greatest importance. Even if the fracture is already several months old, and if there are still sinuses with sequestra between the fragments, it is obvious that the gap is filled with hyperaemic granulation tissue which has not yet undergone fibrosis. In such a case, after simple sequestrectomy unaccompanied by any scraping away of the valuable granulation tissue, the application of a Winnett Orr plaster will usually determine union of the fracture in three or four months. If, on the other hand, the infection has already recovered and the sinuses have healed without the fracture having been immobilized, the granulation tissue has undergone fibrosis and the fragments are sclerosed. It is now useless to immobilize the fracture without first freshening the surfaces, either by multiple drilling or by bone grafting.

If after such a refreshening operation there should he a recurrent flare of infection, it does not by any means follow that the operation will fail. The flare of infection will itself produce the hyperaemia and decalcification which is a necessary preliminary, and if the fragments are immobilized while the infection subsides the fracture will usually unite.

\section{Conclusions}

1. Non-union of fractures is almost always avoidable, and is a complication entirely within the control of the surgeon.

2. Many physiological and biochemical factors may be concerned in the rate of union of fractures, but the only one factor which is of practical importance in determining non-union is inadequate immobilization. Immobilization may be adequate in two ways. (1) The fracture is allowed movement within the splints or plaster ; rotatory movement is especially inimical to union. (2) Immobilization is not continued for a sufficiently long period. There can be no fixed period of immobilization for any fracture ; the average duration of immobility may be exceeded in occasional cases by many months.

3. Hyperaemic decalcification and ischaemic recalcification of bone must be accepted as pathological facts. The initial traumatic hyperaemia mobilizes calcium salts from the bone ends, but rapidly subsides, and allows recalcification of the young connective tissue to form callus. With final repair and fibrosis the callus consolidates by increased calcification. If the hyperaemia is perpetuated by the trauma of movement there is excessive decalcificationa crack fracture becomes a gap fracture. This is the first stage of non-union. In the final phase of ischaemic fibrosis the surfaces of the fragments undergo sclerosis; this is the second stage of non-union. The two stages of nonunion are distinguishable radiographically. The first is cured by immobilization. In the second, preliminary revascularization is necessary by a drilling or grafting operation.

4. The infected compound fracture is pathologically similar to the simple fracture, except that the initial stage of decalcification is prolonged. If it is immobilized the fracture will usually unite. An old infected fracture which has not been immobilized is in the first stage of non-union so long as the infection is active, but passes into the second stage of non-union after quiescence of the infection. In the first stage, sequestrectomy without "scraping," followed by immobilization, determines union. In the second stage a revascularizing operation is necessary. Even if the operation is followed by a flare of infection the fracture still unites if it is immobilized.

\section{Reference}

${ }^{1}$ Watson Jones and Roberts: "Calcification, Decalcification, and Ossification," Brit. Journ. of Surg., xxi, No. 83, p. 461.

E. A. Fennell (Journ. Amer. Med. Assoc., April 7th, 1934) states that at irregular intervals during the last ten years sporadic cases clinically resembling typhoid or paratyphoid fever, but with persistently negative laboratory results, have been reported in Honolulu. The symptoms consisted in a prodromal period; rather sudden onset; rose spots of exaggerated character on chest, abdomen, and inner aspect of the arms and thighs; headache ; cough; and fever. McCoy's suggestion, in 1932, that these might be cases of typhus fever was confirmed by a positive Weil-Felix reaction. Up to date ten cases of endemic typhus have been detected in Honolulu. It differs from epidemic typhus, first, in being essentially a disease of rats, transmitted to man by the flea; secondly, in being a disease of the summer and autumn (whereas epidemic typhus is a disease of winter) ; and thirdly, by its very low fatality rate. 